Proceedings of the Edinburgh Mathematical Society (2003) 46, 383-394 (C)

DOI:10.1017/S0013091501000372 Printed in the United Kingdom

\title{
STABILITY OF THE DEFICIENCY INDICES OF SYMMETRIC OPERATORS UNDER SELF-ADJOINT PERTURBATIONS
}

\author{
EDWARD KISSIN \\ Department of Computing, Communications Technology and Mathematics, \\ London Metropolitan University, 166-220 Holloway Road, \\ London N7 8DB, UK (e.kissin@londonmet.ac.uk)
}

(Received 27 March 2001)

\begin{abstract}
Let $S$ and $T$ be symmetric unbounded operators. Denote by $\overline{S+T}$ the closure of the symmetric operator $S+T$. In general, the deficiency indices of $\overline{S+T}$ are not determined by the deficiency indices of $S$ and $T$. The paper studies some sufficient conditions for the stability of the deficiency indices of a symmetric operator $S$ under self-adjoint perturbations $T$. One can associate with $S$ the largest closed *-derivation $\delta_{S}$ implemented by $S$. We prove that if the unitary operators $\exp (\mathrm{i} t T)$, for $t \in \mathbb{R}$, belong to the domain of $\delta_{S}$ and $\delta_{S}(\exp (\mathrm{i} t T)) \rightarrow 0$ in the strong operator topology as $t \rightarrow 0$, then the deficiency indices of $S$ and $\overline{S+T}$ coincide. In particular, this holds if $S$ and $\exp (\mathrm{i} t T)$ commute or satisfy the infinitesimal Weyl relation.

We also study the case when $S$ and $T$ anticommute: $\exp (-\mathrm{i} t T) S \subseteq S \exp (\mathrm{i} t T)$, for $t \in \mathbb{R}$. We show that if the deficiency indices of $S$ are equal, or if the group $\{\exp (\mathrm{i} t T): t \in \mathbb{R}\}$ of unitary operators has no stationary points in the deficiency space of $S$, then $S$ has a self-adjoint extension which anticommutes with $T$, the operator $S+T$ is closed and the deficiency indices of $S$ and $S+T$ coincide.
\end{abstract}

Keywords: symmetric operator; self-adjoint operator; deficiency indices

2000 Mathematics subject classification: Primary 47B25

\section{Introduction}

Let $S$ be a closed symmetric operator on a Hilbert space $H$ with dense domain $D(S)$ and let $S^{*}$ be its adjoint. The deficiency spaces of $S$

$$
N_{ \pm}(S)=\left\{x \in D\left(S^{*}\right): S^{*} x= \pm \mathrm{i} x\right\}
$$

are closed in $H$ and the numbers $n_{ \pm}(S)=\operatorname{dim}\left(N_{ \pm}(S)\right)$ are called the deficiency indices of $S$. The operator $S$ is self-adjoint if and only if $n_{+}(S)=n_{-}(S)=0$. Let $T$ be another symmetric operator with $D(S) \bigcap D(T)$ dense in $H$ and let $\overline{S+T}$ be the closure of the symmetric operator $S+T$.

Much work has been done on the study of the linear perturbations $\overline{S+T}$ of symmetric operators $S$ and of the stability of their deficiency indices:

$$
n_{+}(\overline{S+T})=n_{+}(S), \quad n_{-}(\overline{S+T})=n_{-}(S) .
$$

In the classical example when $T$ is bounded, not only (1.1) holds but also $\overline{S+T}=S+T$ (see $[\mathbf{1}]$ ). The main thrust of the study was directed towards the important case when 
$S$ is self-adjoint. Rellich [12] and Kato $[\mathbf{5}, \mathbf{6}]$ proved (1.1) when $T$ is $S$-bounded with the $S$-bound less than or equal to 1 . Putnam [11] showed that $S+T$ is self-adjoint if $S$ and $T$ are commuting positive operators. Vasilescu $[\mathbf{1 3}]$ and Pedersen $[\mathbf{1 0}]$ established that $S+T$ is self-adjoint when $S$ and $T$ are self-adjoint and anticommute: $\exp (-\mathrm{i} t T) S \subseteq$ $S \exp (\mathrm{i} t T)$, for $t \in \mathbb{R}$. Numerous applications of these results to differential operators, to the quantum field theory and to the theory of derivations of $C^{*}$-algebras were considered in $[\mathbf{2}, \mathbf{3}, \mathbf{6}-\mathbf{9}, \mathbf{1 1}]$.

In our paper we concentrate on the study of the stability of the deficiency indices of symmetric operators under self-adjoint perturbations, that is, when (1.1) holds if $S$ is symmetric and $T$ is self-adjoint. Unlike the case when both $S$ and $T$ are self-adjoint and the Spectral Theorem can be employed to study the stability, in our case the most suitable tool for the purpose is the theory of indefinite metric spaces: Krein spaces. Using it in Proposition 3.1, we link the deficiency indices of $S$ and $\overline{S+T}$ when both $S$ and $T$ are symmetric. This leads to our first main result (Theorem 4.3), which can be stated in terms of the largest derivation $\delta_{S}$ on $B(H)$ associated with $S$ as follows: (1.1) holds if the group $\{\exp (\mathrm{it} T): t \in \mathbb{R}\}$ of unitary operators lies in the domain of $\delta_{S}$ and $\delta_{S}(\exp (\mathrm{it} T)) x \rightarrow 0$, as $t \rightarrow 0$ for $x \in D\left(S^{*}\right)$. This is a natural generalization of the condition that $S$ and $\exp (\mathrm{it} T)$ commute and it shows, in particular, that (1.1) holds if $\exp (\mathrm{it} T)$ and $S$ satisfy the infinitesimal Weyl relation (4.5). It also points to a link between the theory of perturbation of symmetric operators and the theory of derivations of $C^{*}$-algebras.

Our second main result-Theorems 5.3 and 5.4-is the extension of the results of Pedersen and Vasilescu about anticommuting operators to the case when $S$ is symmetric. Here, again, using the theory of Krein spaces, we show that if the deficiency indices of $S$ are finite and equal, or if the group $\{\exp (\mathrm{it} T): t \in \mathbb{R}\}$ has no stationary points in $N_{+}(S)+N_{-}(S)$, then the operator $S+T$ is closed, (1.1) holds and $S$ has a self-adjoint extension which anticommutes with $T$.

The above results allow us to extend further the conditions under which the index of the ${ }^{*}$-derivation $\delta_{S}$ (see [9]) is stable: making use of Example 36.3 from [9], we conclude that if $S$ is a maximal symmetric operator, then the index of $\delta_{S}$ is stable: $\operatorname{ind}\left(\delta_{S}\right)=$

$\operatorname{ind}\left(\delta_{S+T}\right)$, under any self-adjoint perturbation $T$ such that $S$ and $T$ satisfy conditions of Theorems 4.3 or 5.3 or 5.4 .

\section{Preliminaries}

Let $F$ be a closed, densely defined operator on $H$. Its domain $D(F)$ becomes a Hilbert space with respect to the scalar product

$$
\langle x, y\rangle_{F}=(x, y)+(F x, F y), \quad \text { for } x, y \in D(F) .
$$

Lemma 2.1.

(i) A subset $\Omega$ in $D(F)$ is dense in $\left(D(F),\langle\cdot, \cdot\rangle_{F}\right)$ if and only if $\overline{\left(\left.F\right|_{\Omega}\right)}=F$.

(ii) Let $A$ be a bounded operator and $A D(F) \subseteq D(F)$. Then $\tilde{A}=\left.A\right|_{D(F)}$ is a bounded operator on $\left(D(F),\langle\cdot, \cdot\rangle_{F}\right)$. 
Proof. Part (i) is evident. If $x_{n} \rightarrow x$ and $\tilde{A} x_{n} \rightarrow y$ in $\|\cdot\|_{F}$, they also converge in $\|\cdot\|$, so $y=A x=\tilde{A} x$ and $\tilde{A}$ is closed. By the Closed Graph Theorem, $\tilde{A}$ is bounded.

Let $\boldsymbol{T}=\{T(t): t \geqslant 0\}$ be a strongly continuous one-parameter semigroup of bounded operators on $H: T(0)=\mathbf{1}, T(t+s)=T(t) T(s)$, for $0 \leqslant t, s<\infty$, and

$$
\|T(t) x-x\| \rightarrow 0, \quad \text { as } t \rightarrow 0, \quad \text { for } x \in H .
$$

Its generator $T$ is a closed operator with dense domain $D(T)$ (see [3, Chapter VIII, $\S 1]$ ) and

$$
T(t) D(T) \subseteq D(T) \text { and }\left.\quad T T(t)\right|_{D(T)}=\left.T(t) T\right|_{D(T)}, \quad \text { for } t \geqslant 0 .
$$

Proposition 2.2. Let $T$ be the generator of $\boldsymbol{T}$. If $F$ is a closed operator such that

$$
T(t) D(F) \subseteq D(F), \quad \text { for } t>0 ;
$$

and

$$
\|F T(t) x-F x\| \rightarrow 0, \quad \text { as } t \rightarrow 0, \quad \text { for } x \in D(F),
$$

then $D(F) \bigcap D(T)$ is dense in $\left(D(F),\langle\cdot, \cdot\rangle_{F}\right)$.

Proof. Set $\tilde{T}(t)=\left.T(t)\right|_{D(F)}$. By Lemma $2.1, \tilde{\boldsymbol{T}}=\{\tilde{T}(t): t \geqslant 0\}$ is a one-parameter semigroup of bounded operators on $\left(D(F),\langle\cdot, \cdot\rangle_{F}\right)$. We have

$$
\|\tilde{T}(t) x-x\|_{F}^{2}=\|T(t) x-x\|^{2}+\|F T(t) x-F x\|^{2} \rightarrow 0, \quad \text { as } t \rightarrow 0,
$$

for $x \in D(F)$, so $\tilde{\boldsymbol{T}}$ is strongly continuous. Hence the domain $D(\tilde{T})$ of its generator is dense in $\left(D(F),\langle\cdot, \cdot\rangle_{F}\right)$. Since $D(F) \bigcap D(T)$ contains $D(\tilde{T})$, it is dense in $\left(D(F),\langle\cdot, \cdot\rangle_{F}\right)$.

Let $S$ be a closed symmetric operator and let $S^{*}$ be its adjoint. With respect to $\langle\cdot, \cdot\rangle_{S^{*}}$ (see [3, Chapter XII, $\S 4]), D\left(S^{*}\right)$ is the orthogonal sum of the subspaces $D(S), N_{+}(S)$ and $N_{-}(S)$ :

$$
D\left(S^{*}\right)=D(S)\langle+\rangle_{S^{*}} N_{+}(S)\langle+\rangle_{S^{*}} N_{-}(S) .
$$

Define the following indefinite form on $D\left(S^{*}\right)$ (see $[\mathbf{9}, \S 28]$ ):

$$
[x, y]_{S}=-\mathrm{i}\left\{\left(S^{*} x, y\right)-\left(x, S^{*} y\right)\right\}, \quad \text { for } x, y \in D\left(S^{*}\right) .
$$

It is degenerate on $D(S):[x, y]_{S}=0$ if $x \in D(S)$ and $y \in D\left(S^{*}\right)$, and $[x, y]_{S}=\overline{[y, x]}_{S}$ if $x, y \in D\left(S^{*}\right)$. It is easy to check that

$$
[x, y]_{S}= \begin{cases}\langle x, y\rangle_{S^{*}}=2(x, y), & \text { if } x, y \in N_{+}(S), \\ -\langle x, y\rangle_{S^{*}}=-2(x, y), & \text { if } x, y \in N_{-}(S), \\ 0, & \text { if } x \in N_{-}(S) \text { and } y \in N_{+}(S)\end{cases}
$$

Set

$$
N(S)=N_{+}(S)\langle+\rangle_{S^{*}} N_{-}(S)
$$


With respect to the form $[\cdot, \cdot]_{S}, N(S)$ is a non-degenerate indefinite metric space: for any $z \in N(S)$, there is $u \in N(S)$ such that $[z, u]_{S} \neq 0$. The subspaces $N_{+}(S)$ and $N_{-}(S)$ are, respectively, uniformly positive and uniformly negative. We also have that, for $x, y \in$ $D\left(S^{*}\right)$,

$$
\left|[x, y]_{S}\right| \leqslant\left\|S^{*} x\right\|\|y\|+\|x\|\left\|S^{*} y\right\| \leqslant 2\|x\|_{S^{*}}\|y\|_{S^{*}} .
$$

\section{Sums of symmetric operators}

Let $S$ and $T$ be closed symmetric operators. Set

$$
D=D(S) \bigcap D(T) \text { and } \quad D^{*}=D\left(S^{*}\right) \bigcap D(T) .
$$

We assume that $D$ is dense in $H$ and set $R=\overline{S+T}$. Then $D \subseteq D^{*} \subseteq D\left(R^{*}\right),\left.R\right|_{D}=S+T$ and $\left.R^{*}\right|_{D^{*}}=S^{*}+T$. If $x, y \in D^{*}$, then

$$
[x, y]_{R}=-\mathrm{i}\left\{\left(R^{*} x, y\right)-\left(x, R^{*} y\right)\right\}=-\mathrm{i}\left\{\left(S^{*} x, y\right)-\left(x, S^{*} y\right)\right\}=[x, y]_{S} .
$$

Proposition 3.1. If $\overline{\left(\left.S^{*}\right|_{D^{*}}\right)}=S^{*}$, then $n_{+}(S) \leqslant n_{+}(R)$ and $n_{-}(S) \leqslant n_{-}(R)$.

Proof. Let $\left\{e_{i}\right\}$ be an orthonormal basis in $N_{+}(S)$. By (2.3), $\left\langle e_{i}, e_{j}\right\rangle_{S^{*}}=\left[e_{i}, e_{j}\right]_{S}=$ $\delta_{i j}$. Choose $k \leqslant n_{+}(S)$. Since $D^{*}$ is dense in $\left(D\left(S^{*}\right),\langle\cdot, \cdot\rangle_{S^{*}}\right)$, for any $\epsilon>0$, there are $\left\{h_{i}\right\}_{i=1}^{k}$ in $D^{*}$ such that $\left\|e_{i}-h_{i}\right\|_{S^{*}} \leqslant \epsilon$, for $i=1, \ldots, k$. By (2.4),

$$
\begin{aligned}
\left|\left[h_{i}, h_{j}\right]_{S}-\delta_{i j}\right| & =\left|\left[h_{i}, h_{j}\right]_{S}-\left[e_{i}, e_{j}\right]_{S}\right| \\
& =\left|\left[h_{i}-e_{i}, h_{j}-e_{j}\right]_{S}+\left[e_{i}, h_{j}-e_{j}\right]_{S}+\left[h_{i}-e_{i}, e_{j}\right]_{S}\right| \\
& \leqslant 2\left\|h_{i}-e_{i}\right\|_{S^{*}}\left\|h_{j}-e_{j}\right\|_{S^{*}}+2\left\|e_{i}\right\|_{S^{*}}\left\|h_{j}-e_{j}\right\|_{S^{*}}+2\left\|h_{i}-e_{i}\right\|_{S^{*}}\left\|e_{j}\right\|_{S^{*}} \\
& \leqslant 2 \epsilon^{2}+4 \epsilon \\
& \leqslant 6 \epsilon .
\end{aligned}
$$

Let $x=\sum_{i=1}^{k} \lambda_{i} h_{i}$ for $\lambda_{i} \in \mathbb{C}$. Then $[x, x]_{S}=\sum_{i, j=1}^{k} \lambda_{i} \overline{\lambda_{j}}\left[h_{i}, h_{j}\right]_{S}$. Choosing sufficiently small $\epsilon$ and applying the Principal Minor Test to the matrix $\left(\left[h_{i}, h_{j}\right]_{S}\right)$, we obtain from (3.2) that the quadratic form $[x, x]_{S}$ is positive definite. Hence all $h_{i}$ are linearly independent and the subspace $M$ spanned by $\left\{h_{i}\right\}_{i=1}^{k}$ is positive in $D\left(S^{*}\right)$, that is, $[x, x]_{S}>0$, for $x \in M$. By (3.1), $M$ is also positive in $D\left(R^{*}\right)$.

Let $Q$ be the projection on the subspace $N(R)$ in $\left(D\left(R^{*}\right),\langle\cdot, \cdot\rangle_{R^{*}}\right)$. By $(2.3)$,

$$
[Q x, Q y]_{R}=[x, y]_{R}, \quad \text { for } x, y \in D\left(R^{*}\right) .
$$

Hence $Q M$ is a positive subspace in $N(R)$ and $\operatorname{dim}(Q M)=k$. It follows from the Law of Inertia for indefinite metric spaces (see [9, Corollary 1.12]) that the dimensions of all positive subspaces in $N(R)$ are less than or equal to $\operatorname{dim}\left(N_{+}(R)\right)$. Thus $k \leqslant n_{+}(R)$. Since $k$ is arbitrary, we have $n_{+}(S) \leqslant n_{+}(R)$. Similarly, $n_{-}(S) \leqslant n_{-}(R)$.

The condition $\overline{\left(\left.S^{*}\right|_{D^{*}}\right.}=S^{*}$ in Proposition 3.1 is sufficient but not necessary for $n_{+}(S) \leqslant n_{+}(R)$ and $n_{-}(S) \leqslant n_{-}(R)$. Indeed, if $T=S \neq S^{*}$ then $R=2 S$ and $n_{ \pm}(S)=n_{ \pm}(R)$. However, we have $D^{*}=D\left(S^{*}\right) \bigcap D(S)=D(S)$ and $\overline{\left(\left.S^{*}\right|_{D^{*}}\right)}=S \neq S^{*}$.

We omit the standard proof of the following lemma. 


\section{Lemma 3.2.}

(i) If $\overline{\left(\left.S\right|_{D}\right)}=S$, then $D^{*}=D\left(R^{*}\right) \bigcap D(T)$.

(ii) If $\overline{\left(\left.S^{*}\right|_{D^{*}}\right)}=S^{*}$, then $D=D(R) \bigcap D(T)$.

Proposition 3.1 and Lemma 3.2 yield the following corollary.

Corollary 3.3. If $\overline{\left(\left.R^{*}\right|_{D^{*}}\right)}=R^{*}$ and $\overline{\left(\left.S^{*}\right|_{D^{*}}\right.}=S^{*}$, then $n_{ \pm}(S)=n_{ \pm}(R)$.

\section{Sum of a symmetric operator and the generator of a one-parameter group}

We start with the following result.

Lemma 4.1. Let $\Delta$ be a linear manifold in $D(S)$ such that $\overline{\left(\left.S\right|_{\Delta}\right)}=S$. Let $A$ and $B$ be bounded operators such that $A \Delta \subseteq D(S), B^{*} \Delta \subseteq D(S)$ and let $\left.(S A-B S)\right|_{\Delta}$ extend to a bounded operator $K$. Then $A$ and $B^{*}$ preserve $D(S)$ and $D\left(S^{*}\right)$ and

$$
\left.K\right|_{D\left(S^{*}\right)}=\left.\left(S^{*} A-B S^{*}\right)\right|_{D\left(S^{*}\right)} .
$$

Proof. Let $x \in D(S)$. Since $\overline{\left(\left.S\right|_{\Delta}\right)}=S$, there are $x_{n} \in \Delta$ such that $x_{n} \rightarrow x$ and $S x_{n} \rightarrow S x$. Then $A x_{n} \rightarrow A x$ and $S A x_{n}=B S x_{n}+K x_{n} \rightarrow B S x+K x$. Since $A x_{n} \in D(S)$ and $S$ is closed, $A x \in D(S)$ and $\left.(S A-B S)\right|_{D(S)}=K$. Thus $A$ preserves $D(S)$.

For $x, y \in \Delta$,

$$
\left(K^{*} x, y\right)=(x, K y)=(x,(S A-B S) y)=\left(\left(A^{*} S-S B^{*}\right) x, y\right) .
$$

Since $\Delta$ is dense in $H,\left.K^{*}\right|_{\Delta}=\left.\left(A^{*} S-S B^{*}\right)\right|_{\Delta}$. Repeating the argument used above, we prove that $B^{*} D(S) \subseteq D(S)$ and $\left.K^{*}\right|_{D(S)}=\left.\left(A^{*} S-S B^{*}\right)\right|_{D(S)}$.

Let $y \in D\left(S^{*}\right)$. For any $x \in D(S)$,

$$
(S x, A y)=\left(A^{*} S x, y\right)=\left(K^{*} x, y\right)+\left(S B^{*} x, y\right)=(x, K y)+\left(x, B S^{*} y\right) .
$$

Hence $A y \in D\left(S^{*}\right)$ and (4.1) holds. Similarly, $B^{*}$ preserves $D\left(S^{*}\right)$.

Let $S$ and $T$ be symmetric operators and let i $T$ be the generator of a strongly continuous one-parameter semigroup $\{T(t): t \geqslant 0\}$ of bounded operators.

Proposition 4.2. Let $T(t) D(S) \subseteq D(S)$ and let there exist a family of bounded operators $\{A(t): t>0\}$ on $H$ such that

(1) $A(t)^{*} D(S) \subseteq D(S)$, for $t>0$;

(2) $A(t) x \rightarrow x$, as $t \rightarrow 0$, for $x \in H$;

(3) $\left.(S T(t)-A(t) S)\right|_{D(S)}$ extends to a bounded operator $K(t)$, for $t>0$;

(4) $K(t) x \rightarrow 0$, as $t \rightarrow 0$, for any $x \in D\left(S^{*}\right)$. 
Then

(i) the linear manifolds $D=D(S) \bigcap D(T)$ and $D^{*}=D\left(S^{*}\right) \bigcap D(T)$ are dense in $H$;

(ii) $\overline{\left(\left.S\right|_{D}\right)}=S$ and $\overline{\left(\left.S^{*}\right|_{D^{*}}\right)}=S^{*}$;

(iii) $D(\overline{S+T}) \bigcap D(T)=D$;

(iv) $n_{+}(S) \leqslant n_{+}(\overline{S+T})$ and $n_{-}(S) \leqslant n_{-}(\overline{S+T})$.

Proof. For $x \in D(S)$,

$$
\|S T(t) x-S x\|=\|(A(t) S x+K(t) x)-S x\| \leqslant\|A(t) S x-S x\|+\|K(t) x\| \rightarrow 0,
$$

as $t \rightarrow 0$. By Proposition 2.2, D is dense in $\left(D(S),\langle\cdot, \cdot\rangle_{S}\right)$, so it is dense in $H$.

It follows from Lemma 4.1 that $T(t) D\left(S^{*}\right) \subseteq D\left(S^{*}\right)$, for $t>0$, and

$$
\begin{aligned}
\left\|S^{*} T(t) x-S^{*} x\right\| & =\left\|\left(A(t) S^{*} x+K(t) x\right)-S^{*} x\right\| \\
& \leqslant\left\|A(t) S^{*} x-S^{*} x\right\|+\|K(t) x\| \rightarrow 0,
\end{aligned}
$$

as $t \rightarrow 0$, for $x \in D\left(S^{*}\right)$. Hence, by Proposition 2.2 , the manifold $D^{*}$ is dense in $\left(D\left(S^{*}\right),\langle\cdot, \cdot\rangle_{S^{*}}\right)$, so it is dense in $H$. Part (i) is proved. Part (ii) follows from Lemma 2.1. Part (iii) follows from (ii) and Lemma 3.2, and part (iv) follows from (ii) and Proposition 3.1.

By Stone's Theorem, $T$ is self-adjoint if and only if iT is the generator of a strongly continuous group $\{T(t): t \in \mathbb{R}\}$ of unitary operators: $T(t)=\exp (\mathrm{i} t T)$.

Theorem 4.3. Let $T$ be a self-adjoint operator. Let

(i) $T(t) D(S) \subseteq D(S)$ for each $t \in \mathbb{R}$;

(ii) $\left.(S T(t)-T(t) S)\right|_{D(S)}$ extends to a bounded operator $K(t)$ for each $t \in \mathbb{R}$;

(iii) $K(t) x \rightarrow 0$, as $t \rightarrow 0$, for each $x \in D\left(S^{*}\right)$.

Then $n_{+}(S)=n_{+}(\overline{S+T})$ and $n_{-}(S)=n_{-}(\overline{S+T})$.

Proof. Since $T(t)^{*}=T(-t)$, we obtain from Proposition 4.2 that

$$
D(R) \bigcap D(T)=D, \quad S=\overline{\left(\left.S\right|_{D}\right)}, \quad n_{+}(S) \leqslant n_{+}(R) \quad \text { and } \quad n_{-}(S) \leqslant n_{-}(R) .
$$

It follows from $(2.2)$ that $T(t) D \subseteq D$, for $t \in \mathbb{R}$. Hence

$$
\left.(R T(t)-T(t) R)\right|_{D}=\left.(S T(t)-T(t) S)\right|_{D}+\left.(T T(t)-T(t) T)\right|_{D}=\left.K(t)\right|_{D} .
$$

Since $R=\overline{\left(\left.R\right|_{D}\right)}$, it follows from Lemma 4.1 that

$$
T(t) D(R) \subseteq D(R) \quad \text { and }\left.\quad(R T(t)-T(t) R)\right|_{D(R)}=\left.K(t)\right|_{D(R)} .
$$


The operator $-\mathrm{i} T$ is the generator of the group $\{T(-t): t \in \mathbb{R}\}$ and, by (4.3), the group and the operator $R$ satisfy the conditions of Proposition 4.2. Let $W$ be the closure of the operator $\left.(R-T)\right|_{D}$. We obtain from (4.2) and from Proposition 4.2 that

$$
D(W) \bigcap D(T)=D \quad \text { and } \quad n_{+}(R) \leqslant n_{+}(W), \quad n_{-}(R) \leqslant n_{-}(W) .
$$

Since $\left.W\right|_{D}=\left.(R-T)\right|_{D}=\left.(S+T-T)\right|_{D}=\left.S\right|_{D}$, it follows from (4.2) that $W=\overline{\left(\left.W\right|_{D}\right)}=\overline{\left(\left.S\right|_{D}\right)}=S$. Comparing (4.2) and (4.4), we have $n_{+}(S)=n_{+}(R)$ and $n_{-}(S)=n_{-}(R)$.

A self-adjoint operator $T$ and a symmetric operator $S$ commute if

$$
\exp (\mathrm{it} T) D(S) \subseteq D(S) \quad \text { and }\left.\quad S \exp (\mathrm{i} t T)\right|_{D(S)}=\left.\exp (\mathrm{i} t T) S\right|_{D(S)}, \quad \text { for } t \in \mathbb{R} .
$$

They satisfy the infinitesimal Weyl relation (see [4]) if $\exp (\mathrm{i} t T) D(S) \subseteq D(S)$ and

$$
\left.(S \exp (\mathrm{i} t T)-\exp (\mathrm{i} t T) S)\right|_{D(S)}=\left.t \exp (\mathrm{i} t T)\right|_{D(S)}, \quad \text { for } t \in \mathbb{R} .
$$

Corollary 4.4. Let $S$ be a symmetric operator and $T$ be a self-adjoint operator. If $T$ and $S$ commute or satisfy the infinitesimal Weyl relation (4.5), then

$$
n_{+}(\overline{S+T})=n_{+}(S) \text { and } n_{-}(\overline{S+T})=n_{-}(S) .
$$

Even if $S$ is self-adjoint and commutes with $\boldsymbol{T}(T)$, the operator $S+T$ is not necessarily closed. If, for example, $T=-S$, then $S+T=\left.\mathbf{0}\right|_{D(S)}$ is not closed. Putnam [11] showed that if $S$ and $T$ are positive and commute, then $S+T$ is self-adjoint.

\section{Anticommuting operators}

A self-adjoint operator $T$ and a symmetric operator $S$ anticommute (cf. $[\mathbf{1 0}, \mathbf{1 3}])$ if $\exp (\mathrm{i} t T) D(S) \subseteq D(S)$ and

$$
\left.S \exp (\mathrm{i} t T)\right|_{D(S)}=\left.\exp (-\mathrm{i} t T) S\right|_{D(S)}, \quad \text { for } t \in \mathbb{R} .
$$

For self-adjoint $S$, Vasilescu [13] and Pedersen [10] proved that the operator $S+T$ is closed and self-adjoint. We study the case when $S$ is symmetric.

We have from Proposition 4.2 that, for any anticommuting operators $S$ and $T$, $n_{+}(S) \leqslant n_{+}(\overline{S+T})$ and $n_{-}(S) \leqslant n_{-}(\overline{S+T})$. Hence if $n_{+}(S)=n_{-}(S)=\infty$, then

$$
n_{+}(S)=n_{+}(\overline{S+T}) \quad \text { and } \quad n_{-}(S)=n_{-}(\overline{S+T}) .
$$

We will extend this to all symmetric $S$. Set $T(t)=\exp (\mathrm{i} t T)$. From Lemma 4.1 we have

$$
T(t) D\left(S^{*}\right) \subseteq D\left(S^{*}\right) \quad \text { and }\left.\quad S^{*} T(t)\right|_{D\left(S^{*}\right)}=\left.T(-t) S^{*}\right|_{D\left(S^{*}\right)}, \quad \text { for } t \in \mathbb{R} .
$$

Set $\tilde{T}(t)=\left.T(t)\right|_{D\left(S^{*}\right)}$. As in Proposition $2.2,\{\tilde{T}(t): t \in \mathbb{R}\}$ is a strongly continuous oneparameter group of bounded operators on $\left(D\left(S^{*}\right),\langle\cdot, \cdot\rangle_{S^{*}}\right)$. 
Lemma 5.1. All operators $\tilde{T}(t)$ are unitary and preserve $N(S)$.

Proof. Since all $T(t)$ are unitary, it follows from (5.2) that

$$
\begin{aligned}
\langle\tilde{T}(t) x, \tilde{T}(t) y\rangle_{S^{*}} & =(T(t) x, T(t) y)+\left(S^{*} T(t) x, S^{*} T(t) y\right) \\
& =(x, y)+\left(T(-t) S^{*} x, T(-t) S^{*} y\right)=(x, y)+\left(S^{*} x, S^{*} y\right)=\langle x, y\rangle_{S^{*}},
\end{aligned}
$$

for $x, y \in D\left(S^{*}\right)$. Hence all operators $\tilde{T}(t)$ are unitary. Since they preserve the subspace $D(S)$ of $\left(D\left(S^{*}\right),\langle\cdot, \cdot\rangle_{S^{*}}\right)$, they also preserve its orthogonal complement $N(S)$.

Set $U(t)=\left.\tilde{T}(t)\right|_{N(S)}$ and $J=-\left.\mathrm{i} S^{*}\right|_{N(S)}$. Then $\boldsymbol{U}=\{U(t): t \in \mathbb{R}\}$ is a strongly continuous one-parameter group of unitary operators on the Hilbert space $\left(N(S),\langle\cdot, \cdot\rangle_{S^{*}}\right)$,

$$
J x=x \quad \text { if } x \in N_{+}(S), \quad J x=-x \quad \text { if } x \in N_{-}(S),
$$

and

$$
J U(t)=U(-t) J, \quad \text { for } t \in \mathbb{R} .
$$

Let $\mathrm{i} W$ be the generator of the group $\boldsymbol{U}$. Then $W$ is a self-adjoint operator on $N(S)$ and the operators $W$ and $J$ anticommute (see (5.1)). It follows from Proposition 1.1 in [10] that

$$
J D(W) \subseteq D(W),\left.\quad J W\right|_{D(W)}=-\left.W J\right|_{D(W)} .
$$

Since $J^{2}=\mathbf{1}_{N(S)}$, we have $J D(W)=D(W)$. Set $D_{ \pm}=D(W) \bigcap N_{ \pm}(S)$.

\section{Lemma 5.2.}

(i) The linear manifolds $D_{+}$and $D_{-}$are dense in $N_{+}(S)$ and $N_{-}(S)$, respectively,

$$
W D_{+} \subseteq N_{-}(S), \quad W D_{-} \subseteq N_{+}(S) \quad \text { and } \quad D(W)=D_{+}\langle+\rangle_{S^{*}} D_{-} .
$$

(ii) If one of the deficiency indices of $S$ is finite, then $D_{+}=N_{+}(S), D_{-}=N_{-}(S), W$ is a bounded operator and the group $\boldsymbol{U}$ is uniformly continuous.

Proof. If $x \in D_{+}$, then $J(W x)=-W J x=-W x$, so $W x \in N_{-}(S)$. Thus $W D_{+} \subseteq N_{-}(S)$. Similarly, $W D_{-} \subseteq N_{+}(S)$. If $x \in D(W)$, then $J x \in D(W)$ and $J(x+J x)=x+J x$, so that $x+J x \in D(W) \bigcap N_{+}(S)=D_{+}$. Similarly, $x-J x \in D_{-}$. Thus $D(W)=D_{+}\langle+\rangle_{S^{*}} D_{-}$. Since $D(W)$ is dense in $N(S), D_{-}$is dense in $N_{-}(S)$ and $D_{+}$is dense in $N_{+}(S)$.

Assume now that $n_{+}(S)<\infty$. Since $D_{+}$is dense in $N_{+}(S)$, we have $D_{+}=N_{+}(S)$. Set

$$
K_{ \pm}=\left\{x \in D_{ \pm}: W x=0\right\} .
$$

Since $W D_{-} \subseteq D_{+}$, we have $\operatorname{dim}\left(W D_{-}\right) \leqslant n_{+}(S)<\infty$, so the quotient space $D_{-} / K_{-}$is finite dimensional. Since $W$ is closed and $D_{-}$is dense in $N_{-}(S)$, we have $D_{-}=N_{-}(S)$. Thus $D(W)=N(S)$ and $W$ is bounded. By Corollary VIII.1.9 in [3], the group $\boldsymbol{U}$ is uniformly continuous. 
Let $L$ be a subspace of $N(S)$. Its $[\cdot, \cdot]_{S}$-orthogonal 'complement' is defined by

$$
L^{[\perp]}=\left\{y \in N(S):[x, y]_{S}=0, \text { for } x \in L\right\} .
$$

$L$ is called neutral if $L \subseteq L^{[\perp]}$, that is, $[x, y]_{S}=0$, for $x, y \in L$. It is maximal neutral if it is not contained in any larger neutral subspace.

Similarly, a subspace $\mathcal{L}$ of $D\left(S^{*}\right)$ is neutral if $[x, y]_{S}=0$ for $x, y \in \mathcal{L}$. If $L$ is a neutral space in $N(S)$ then, by $(2.3), \mathcal{L}=D(S)\langle+\rangle_{S^{*}} L$ is a neutral space in $D\left(S^{*}\right)$. The operator $\tilde{S}=\left.S^{*}\right|_{\mathcal{L}}$ is a symmetric extension of $S$ (see $[\mathbf{3}, \S$ XII.4]) and

$$
n_{+}(\tilde{S})=n_{+}(S)-\operatorname{dim}(L) \quad \text { and } \quad n_{-}(\tilde{S})=n_{-}(S)-\operatorname{dim}(L) .
$$

The operator $\tilde{S}$ is self-adjoint, that is, $n_{+}(\tilde{S})=n_{-}(\tilde{S})=0$, if and only if

$$
L=L^{[\perp]} \text {. }
$$

It follows from (2.2) and from the properties of the operator $J$ that

$$
[x, y]_{S}=\langle J x, y\rangle_{S^{*}}, \quad \text { for } x, y \in N(S) .
$$

Theorem 5.3. Let a symmetric operator $S$ and a self-adjoint operator $T$ anticommute. Set $G=\{x \in H: \exp (\mathrm{i} t T) x=x$, for $t \in \mathbb{R}\}$. If

$$
G \bigcap N(S)=\{0\}
$$

then

(i) $n_{-}(S)=n_{+}(S)$;

(ii) $S$ has a self-adjoint extension which anticommutes with $T$;

(iii) if the deficiency indices of $S$ are finite, the operator $S+T$ is closed;

(iv) $n_{+}(S)=n_{+}(\overline{S+T})$ and $n_{-}(S)=n_{-}(\overline{S+T})$.

Proof. Since $W$ is closed, $\operatorname{Ker}(W)$ is closed in $N(S)$. By $(2.2), \operatorname{Ker}(W)$ is invariant for all $U(t)$. Therefore, if $x \in \operatorname{Ker}(W)$, then $\exp (\mathrm{i} t T) x=U(t) x=x$, for $t \in \mathbb{R}$. Since $G \bigcap N(S)=\{0\}$, we have $\operatorname{Ker}(W)=\{0\}$, so that $K_{-}=K_{+}=\{0\}$. If $n_{+}(S)<\infty$, then, by Lemma $5.2, D_{-}=N_{-}(S)$ and $W D_{-} \subseteq N_{+}(S)$. Since $K_{-}=\{0\}$, we have

$$
n_{-}(S)=\operatorname{dim}\left(D_{-}\right)=\operatorname{dim}\left(W D_{-}\right) \leqslant n_{+}(S) .
$$

Similarly, $n_{+}(S)=\operatorname{dim}\left(D_{+}\right)=\operatorname{dim}\left(W D_{+}\right) \leqslant n_{-}(S)$. Part (i) is proved.

Let $E(t)$ be the spectral function of $W$. Since $\operatorname{Ker}(W)=\{0\}$, we have $E(0)=$ $\lim _{t \rightarrow 0+} E(t)$. Set $M=E(0) N(S)$ and $L=\left(\mathbf{1}_{N(S)}-E(0)\right) N(S)$. Then

$$
N(S)=M\langle+\rangle_{S^{*}} L
$$


Since $J$ and $W$ anticommute and since $J$ is bounded, it follows from Proposition 1.4 in $[\mathbf{1 0}]$ that $E(t) J=J\left(\mathbf{1}_{N(S)}-E(-t)\right)$, for $t \in \mathbb{R}$. Thus

$$
J L=J\left(\mathbf{1}_{N(S)}-E(0)\right) N(S)=E(0) J N(S)=E(0) N(S)=M .
$$

Since $J^{2}=\mathbf{1}_{N(S)}$, we have $J M=L$.

If $x \in L$, then $J x \in M$. Making use of (5.8) and (5.10), we obtain that $[x, y]_{S}=$ $\langle J x, y\rangle_{S^{*}}=0$, for $y \in L$, so that $L$ is a neutral subspace. Similarly, $M$ is neutral and, moreover, $L$ and $M$ are maximal neutral subspaces in $N(S)$ and they coincide with their $[\cdot, \cdot]_{S}$-orthogonal 'complements' in $N(S): L^{[\perp]}=L$ and $M^{[\perp]}=M$.

Set $\mathcal{L}=D(S)\langle+\rangle_{S^{*}} L$ and $\tilde{S}=\left.S^{*}\right|_{\mathcal{L}}$. We obtain from (5.6) and (5.7) that $\tilde{S}$ is a selfadjoint extension of $S$ and that

$$
0=n_{+}(\tilde{S})=n_{+}(S)-\operatorname{dim}(L) \quad \text { and } \quad 0=n_{-}(\tilde{S})=n_{-}(S)-\operatorname{dim}(L) .
$$

Since the projection $E(0)$ commutes with all operators $U(t)$, the subspaces $L$ and $M$ are invariant for $U(t)$ with $t \in \mathbb{R}$. Hence

$$
\begin{aligned}
\exp (\mathrm{i} t T) D(\tilde{S}) & =\exp (\mathrm{i} t T) \mathcal{L}=\exp (\mathrm{i} t T)\left(D(S)\langle+\rangle_{S^{*}} L\right) \\
& \subseteq D(S)\langle+\rangle_{S^{*}} U(t) L=D(\tilde{S})
\end{aligned}
$$

and, by (5.2),

$$
\begin{aligned}
\left.\tilde{S} \exp (\mathrm{i} t T)\right|_{D(\tilde{S})} & =\left.S^{*} \exp (\mathrm{i} t T)\right|_{D(\tilde{S})} \\
& =\left.\exp (-\mathrm{i} t T) S^{*}\right|_{D(\tilde{S})}=\left.\exp (-\mathrm{i} t T) \tilde{S}\right|_{D(\tilde{S})} .
\end{aligned}
$$

Thus the operators $\tilde{S}$ and $T$ anticommute. Part (ii) is proved.

Assume now that the deficiency indices of $S$ are finite. It follows from Lemma 5.2 that $N(S) \subset D(T)$. Set $D=D(S) \cap D(T)$. We obtain from Proposition 4.2 that $D(\overline{S+T}) \bigcap D(T)=D$. Since $L \subset N(S) \subset D(T)$ and $L \bigcap D=\{0\}$, we have $D(\overline{S+T}) \bigcap L=\{0\}$.

On the other hand, the operator $\tilde{S}+T$ is defined on

$$
\begin{aligned}
\mathcal{M} & =D(\tilde{S}) \bigcap D(T)=\left(D(S)\langle+\rangle_{S^{*}} L\right) \bigcap D(T) \\
& =(D(S) \bigcap D(T))\langle+\rangle_{S^{*}} L=D\langle+\rangle_{S^{*}} L
\end{aligned}
$$

Since $\tilde{S}$ and $T$ anticommute, it follows from Theorem 2.1 in $[\mathbf{1 0}]$ that $\tilde{S}+T$ is self-adjoint and hence closed: $D(\tilde{S}+T)=\mathcal{M}$. Clearly, $\tilde{S}+T$ is a self-adjoint extension of $S+T$. Thus $S+T \subseteq \overline{S+T} \subseteq \tilde{S}+T$. If $S+T \neq \overline{S+T}$, then $D \subset D(\overline{S+T}) \subseteq D\langle+\rangle_{S^{*}} L$, so $D(\overline{S+T}) \cap L \neq\{0\}$. This contradiction shows that $S+T$ is closed. Part (iii) is proved.

If $n_{ \pm}(S)=\infty$, then (iv) follows from Proposition 4.2. Let $n_{ \pm}(S)$ be finite. By (iii), $R=S+T$ is closed, $D(R)=D$ and $R \subseteq \tilde{S}+T$. Since $\tilde{S}+T$ is self-adjoint, $\tilde{S}+T \subseteq R^{*}$, so that $\tilde{S}+T=\left.R^{*}\right|_{\mathcal{M}}$. Since $L$ is neutral, we have from (2.3) that $\mathcal{M}$ is a neutral subspace in $D\left(S^{*}\right)$. Hence, by $(3.1), \mathcal{M}$ is a neutral subspace in $D\left(R^{*}\right)$. Since 
$D\left(R^{*}\right)=D(R)\langle+\rangle_{R^{*}} N(R)=D\langle+\rangle_{R^{*}} N(R)$, we obtain that $\mathcal{M}=D\langle+\rangle_{R^{*}} L^{\prime}$, where $\operatorname{dim}(L)=\operatorname{dim}\left(L^{\prime}\right)$ and $L^{\prime}$ is a neutral subspace in $N(R)$. By (5.6),

$$
n_{+}\left(\left.R^{*}\right|_{\mathcal{M}}\right)=n_{+}(R)-\operatorname{dim}\left(L^{\prime}\right) \quad \text { and } \quad n_{-}\left(\left.R^{*}\right|_{\mathcal{M}}\right)=n_{-}(R)-\operatorname{dim}\left(L^{\prime}\right) .
$$

Since $\tilde{S}+T=\left.R^{*}\right|_{\mathcal{M}}$ is self-adjoint, we have $n_{+}\left(\left.R^{*}\right|_{\mathcal{M}}\right)=n_{-}\left(\left.R^{*}\right|_{\mathcal{M}}\right)=0$. Therefore,

$$
n_{+}(R)=n_{-}(R)=\operatorname{dim}\left(L^{\prime}\right)=\operatorname{dim}(L) .
$$

Comparing this with (5.12), we complete the proof.

In the next theorem we consider the case when $n_{-}(S)=n_{+}(S)<\infty$ and prove the results of Theorem 5.3 without the restriction given in (5.9).

Theorem 5.4. Let the operators $S$ and $T$ be the same as in Theorem 5.3. If $n_{-}(S)=$ $n_{+}(S)<\infty$, then

(i) $S$ has a self-adjoint extension which anticommutes with $T$;

(ii) the operator $S+T$ is closed;

(iii) $n_{+}(S)=n_{+}(S+T)$ and $n_{-}(S)=n_{-}(S+T)$.

Proof. Since $n_{ \pm}(S)<\infty$, it follows from Lemma 5.2 that $D_{ \pm}=N_{ \pm}(S)$, so $K_{ \pm}=\{x \in$ $\left.N_{ \pm}(S): W x=0\right\}$ (see (5.5)). Let $P_{+}$be the orthogonal complement of $K_{+}$in $N_{+}(S)$ and let $P_{-}$be the orthogonal complement of $K_{-}$in $N_{-}(S)$. By Lemma 5.2, $W P_{+} \subseteq N_{-}(S)$. Since $W$ is self-adjoint, if $y \in P_{+}$and $x \in K_{-}$, then $0=\langle W x, y\rangle_{S^{*}}=\langle x, W y\rangle_{S^{*}}$. Hence $W P_{+} \subseteq P_{-}$. Similarly, $W P_{-} \subseteq P_{+}$. Thus

$$
\operatorname{dim}\left(P_{+}\right)=\operatorname{dim}\left(W P_{+}\right) \leqslant \operatorname{dim}\left(P_{-}\right) \quad \text { and } \quad \operatorname{dim}\left(P_{-}\right)=\operatorname{dim}\left(W P_{-}\right) \leqslant \operatorname{dim}\left(P_{+}\right) .
$$

Therefore, $\operatorname{dim}\left(P_{+}\right)=\operatorname{dim}\left(P_{-}\right)$, so that $\operatorname{dim}\left(K_{+}\right)=\operatorname{dim}\left(K_{-}\right)$.

The subspace $P=P_{+}\langle+\rangle_{S^{*}} P_{-}$is invariant for $W$. If $e$ is an eigenvector of $W$ in $P$ with eigenvalue $\lambda$, it follows from (5.3) that $W(J e)=-J W e=-\lambda J e$, so $J e$ is an eigenvector of $W$ with eigenvalue $-\lambda$. Since $\operatorname{Ker}(W \mid P)=\{0\}$, there is an orthonormal basis $\left\{e_{1}, \ldots, e_{n}, f_{1}, \ldots, f_{n}\right\}$ in $\left(P,\langle\cdot, \cdot\rangle_{S^{*}}\right)$ such that

$$
f_{i}=J e_{i}, \quad W e_{i}=\lambda_{i} e_{i}, \quad W f_{i}=-\lambda_{i} f_{i} \quad \text { and } \quad \lambda_{i}>0 .
$$

We obtain from (5.8) that

$$
\left[e_{i}, e_{j}\right]_{S}=\left\langle J e_{i}, e_{j}\right\rangle_{S^{*}}=\left\langle f_{i}, e_{j}\right\rangle_{S^{*}}=0
$$

for all $i, j$. Thus the subspace $\mathcal{M}$ spanned by all $\left\{e_{i}\right\}_{i=1}^{n}$ is neutral, invariant for $W$ and its $[\cdot, \cdot]_{S}$-orthogonal complement in $P$ coincides with $\mathcal{M}$.

Let $K=K_{+}\langle+\rangle_{S^{*}} K_{-}$, let $m=\operatorname{dim}\left(K_{+}\right)=\operatorname{dim}\left(K_{-}\right)$and let $\left\{h_{i}^{-}\right\}_{i=1}^{m}$ and $\left\{h_{i}^{+}\right\}_{i=1}^{m}$ be orthonormal bases in $K_{-}$and $K_{+}$, respectively. By (5.8),

$$
\begin{aligned}
{\left[h_{i}^{-}+h_{i}^{+}, h_{j}^{-}+h_{j}^{+}\right]_{S} } & =\left\langle J\left(h_{i}^{-}+h_{i}^{+}\right), h_{j}^{-}+h_{j}^{+}\right\rangle_{S^{*}}=\left\langle-h_{i}^{-}+h_{i}^{+}, h_{j}^{-}+h_{j}^{+}\right\rangle_{S^{*}} \\
& =-\left\langle h_{i}^{-}, h_{j}^{-}\right\rangle_{S^{*}}+\left\langle h_{i}^{+}, h_{j}^{+}\right\rangle_{S^{*}}=0,
\end{aligned}
$$


for all $i, j$. Hence the subspace $\mathcal{N}$ spanned by $\left\{h_{i}^{-}+h_{i}^{+}\right\}_{i=1}^{m}$ is neutral and its $[\cdot, \cdot]_{S^{-}}$orthogonal complement in $K$ coincides with $\mathcal{N}$. Since $\mathcal{N} \subset \operatorname{Ker}(W)$, we have that $\mathcal{N}$ is invariant for $W$.

The subspace $L=\mathcal{M}\langle+\rangle_{S^{*}} \mathcal{N}$ is invariant for $W$. Since $J \mathcal{M} \subset P$ and $J \mathcal{N} \subset K$, the subspaces $\mathcal{M}$ and $\mathcal{N}$ are orthogonal with respect to $[\cdot, \cdot]_{S}$. Hence $L$ is neutral and $L^{[\perp]}=L$. Setting $\mathcal{L}=D(S)\langle+\rangle_{S^{*}} L$ and $\tilde{S}=\left.S^{*}\right|_{\mathcal{L}}$ and repeating the argument used in the proof of Theorem 5.3, we complete the proof of the theorem.

Acknowledgements. The author is grateful to the Leverhulme Trust for the award of a research fellowship.

\section{References}

1. N. I. Ahiezer and I. M. Glazman, The theory of linear operators in Hilbert spaces (Ungar, New York, 1961).

2. J. M. Cook, Asymptotic properties of a boson field with a given source, J. Math. Phys. 2 (1961), 33-45.

3. N. Dunford And J. T. Schwartz, Linear operators, vols I and II (Wiley Interscience, New York, 1963).

4. P. E. T. Jorgensen and P. S. Muhly, Self-adjoint extensions satisfying the Weyl operator commutation relations, J. Analyse Math. 37 (1980), 46-99.

5. T. Kato, On the convergence of the perturbation method, J. Fac. Sci. Univ. Tokyo (1) 6 (1951), 145-226.

6. T. Kato, Perturbation theory for linear operators (Springer, 1966).

7. Y. Kato, Some converging examples of the perturbation series in the quantum field theory, Prog. Theor. Phys. 26 (1961), 99-122.

8. Y. Kato and N. Mugibayashi, Regular perturbation and asymptotic limits of operators in quantum field theory, Prog. Theor. Phys. 30 (1963), 103-133.

9. E. Kissin And V. Shulman, Representations on Krein spaces and derivations of $C^{*}$ algebras (Addison-Wesley, 1997).

10. S. Pedersen, Anticommuting self-adjoint operators, J. Funct. Analysis 89 (1990), 428443.

11. C. R. Putnam, Commutation properties of Hilbert space operators (Springer, 1967).

12. F. Rellich, Storungstheorie der Spektralzerlegung, III, Math. Ann. 116 (1939), 555-570.

13. F.-H. VAsilescu, Anticommuting self-adjoint operators, Rev. Roumaine Math. Pures Appl. 28 (1983), 77-91. 